\title{
Exact reformulation of the bosonic many-body problem in terms of stochastic wave functions: convergence issues
}

\author{
Iacopo Carusotto and Yvan Castinf \\ Laboratoire Kastler Brossel, École Normale Supérieure, \\ 24 rue Lhomond, 75231 Paris Cedex 05, France
}

(Dated: November 15, 2018)

\begin{abstract}
There exist methods to reformulate in an exact way the many-body problem of interacting bosons in terms of the stochastic evolution of single particle wave functions. For one such reformulation, the so-called simple Fock scheme, we present an elementary derivation, much simpler than the original one. Furthermore, we show that two other schemes, based on coherent states of the matter field rather than on Fock states, lead to an infinite statistical uncertainty in the continuous time limit. The simple Fock scheme is therefore, up to now, the only one that was proved to lead to a convergent Monte Carlo simulation scheme at all times.

PACS numbers: 05.30.Jp, 03.75.Fi, 02.70.Ss
\end{abstract}

\section{INTRODUCTION}

Consider a gas of $N$ indistinguishable bosonic spinless particles with binary interactions, a situation typically encountered in Bose condensed atomic gases [1]. The time evolution of the many-body state vector $|\psi\rangle$ of the gas is described by the Schrödinger equation

$$
\frac{d}{d t}|\psi\rangle=\frac{1}{i \hbar} \mathcal{H}|\psi\rangle
$$

where $\mathcal{H}$ is the Hamiltonian of the system, including kinetic energy, trapping potential energy and interaction terms. In the general case of a large number of particles and a large number of modes of the atomic matter field, the Hilbert space of the system is far too large for a direct integration of (1) to be feasible on a computer. A possible way out, intensively explored in particular in the quantum optics community, is to reformulate the evolution equation in terms of the stochastic evolution of classical fields, which are much smaller objects. Historically, the first suggestion for such a reformulation is based on the Positive-P distribution [2, 3]. Other schemes have been proposed more recently, based on different representations of the many-body density operator [4, 5, 6]. Generalizations to fermionic systems are also possible $[$, 8].

As these methods are stochastic in nature, their prediction for a finite number of realizations deviates from the exact result by a fluctuating, zero-mean quantity, called the statistical error. One of the challenges of such reformulations is to prove that the average of the absolute value of the statistical error remains finite at all times, so that an actual Monte Carlo simulation converges to the exact result in the limit of an infinite number of realizations ${ }^{1}$. To our knowledge, a proof of such a convergence property for the multi-mode Hamiltonian problem defined above was given

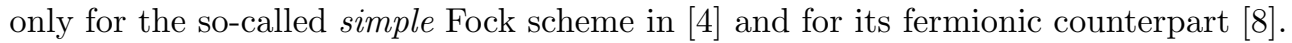

The goal of the present paper is twofold. First, it presents a much simpler derivation of the simple Fock scheme than the original one in 沟: the main idea here is to work directly with state vectors $|\psi\rangle$ rather than with the many-body density operator. Second, it addresses the issue of the convergence of two other schemes based on coherent states of the bosonic field: the simple coherent scheme of [4] and a new scheme that we call the Bargmann scheme. It is found that these two schemes based on coherent states lead to an infinite mean of the absolute value of the statistical error for any finite evolution time.

\footnotetext{
${ }^{1}$ Let us call $\delta$ the absolute value of the statistical error (to be defined more precisely later on). Let us assume that the probability distribution for $\delta$ has a long tail scaling as $1 / \delta^{1+\mu}$ for large $\delta$. The most favorable case is $\mu>2$, in which case $\delta$ has a finite variance and the statistical error for a sample of $\mathcal{N}_{r}$ Monte Carlo realizations scales as $1 / \sqrt{\mathcal{N}_{r}}$. In the intermediate $1<\mu<2$ case, the convergence is slower, scaling as $1 / \mathcal{N}_{r}^{1-1 / \mu}$. In the worst $\mu<1$ case, the mean value of $\delta$ is infinite and the statistical error of the Monte Carlo simulation grows to infinity with the number of realizations as $\mathcal{N}_{r}^{-1+1 / \mu}$. For more details, see [9].
} 


\section{THE MODEL HAMILTONIAN}

A model for an ultracold trapped interacting Bose gas in $D$ dimensions with short range interactions can be obtained in a second-quantization formalism by using the Hamiltonian

$$
\mathcal{H}=\sum_{\mathbf{r}} \Delta V \hat{\Psi}^{\dagger}(\mathbf{r}) h_{0} \hat{\Psi}(\mathbf{r})+\frac{g_{0}}{2} \sum_{\mathbf{r}} \Delta V \hat{\Psi}^{\dagger}(\mathbf{r}) \hat{\Psi}^{\dagger}(\mathbf{r}) \hat{\Psi}(\mathbf{r}) \hat{\Psi}(\mathbf{r}) ;
$$

the spatial coordinate $\mathbf{r}$ runs on a discrete orthogonal lattice of $M$ points with periodic boundary conditions; $V$ is the total volume of the quantization box and $\Delta V=V / M$ is the volume of the unit cell of the lattice. $h_{0}=\frac{p^{2}}{2 m}+U_{\text {ext }}$ is the one-body Hamiltonian in the external trapping potential $U_{\text {ext }}(\mathbf{r}), m$ is the atomic mass and interactions are modeled by a two-body discrete delta potential with a coupling constant $g_{0}$. The field operators $\hat{\Psi}(\mathbf{r})$ satisfy the Bose commutation relations $\left[\hat{\Psi}(\mathbf{r}), \hat{\Psi}^{\dagger}\left(\mathbf{r}^{\prime}\right)\right]=\delta_{\mathbf{r}, \mathbf{r}^{\prime}} / \Delta V$ and can be expanded on plane waves according to $\hat{\Psi}(\mathbf{r})=\sum_{\mathbf{k}} \hat{a}_{\mathbf{k}} e^{i \mathbf{k r}} / \sqrt{V}$ with $\mathbf{k}$ restricted to the first Brillouin zone of the reciprocal lattice; in the plane wave basis, the kinetic energy term has the diagonal form $\sum_{\mathbf{k}} \frac{\hbar^{2} \mathbf{k}^{2}}{2 m} \hat{a}_{\mathbf{k}}^{\dagger} \hat{a}_{\mathbf{k}}$. In order for the discrete model to correctly reproduce the underlying continuous field theory, the grid spacing must be smaller than macroscopic length scales like the thermal de Broglie wavelength and the healing length.

\section{THE SIMPLE FOCK SCHEME}

Consider the $N$ particle bosonic Hartree-Fock state defined as

$$
|N: \phi\rangle=\frac{1}{\sqrt{N !}} \hat{a}_{\phi}^{\dagger N}|0\rangle
$$

where the operator $\hat{a}_{\phi}^{\dagger}$ creates a particle in the non-necessarily normalized single-particle wave function $\phi(\mathbf{r})$ :

$$
\hat{a}_{\phi}^{\dagger}=\sum_{\mathbf{r}} \Delta V \phi(\mathbf{r}) \hat{\Psi}^{\dagger}(\mathbf{r})
$$

The challenge is here to find an equation of motion for $\phi(\mathbf{r})$ in order for the Hartree-Fock ansatz to give an exact solution to the full many-body dynamics, i.e. we aim to obtain a dynamics for $\phi(\mathbf{r})$ such that

$$
d|N: \phi\rangle \stackrel{?}{=} \frac{d t}{i \hbar} \mathcal{H}|N: \phi\rangle
$$

in a sense to be defined. In the following we proceed to evaluate both sides of (5).

The action of the Hamiltonian (2) on $|N: \phi\rangle$ can be worked out by splitting the field operator in its longitudinal and orthogonal components with respect to $\phi$ :

$$
\hat{\Psi}(\mathbf{r})=\frac{\phi(\mathbf{r})}{\|\phi\|^{2}} \hat{a}_{\phi}+\hat{\Psi}_{\perp}(\mathbf{r})
$$

where the squared norm of $\phi$ is

$$
\|\phi\|^{2}=\langle\phi \mid \phi\rangle
$$

with the following scalar product

$$
\langle\phi \mid \psi\rangle=\sum_{\mathbf{r}} \Delta V \phi^{*}(\mathbf{r}) \psi(\mathbf{r})
$$

From this splitting of the field operator it follows that

$$
\begin{aligned}
\hat{\Psi}(\mathbf{r})|N: \phi\rangle & =\sqrt{N} \phi(\mathbf{r})|N-1: \phi\rangle \\
\hat{\Psi}^{\dagger}(\mathbf{r})|N: \phi\rangle & =\sqrt{N+1} \frac{\phi^{*}(\mathbf{r})}{\|\phi\|^{2}}|N+1: \phi\rangle+\hat{\Psi}_{\perp}^{\dagger}(\mathbf{r})|N: \phi\rangle .
\end{aligned}
$$


Inserting these expressions into the Schrödinger equation for the Hartree-Fock state and collecting the terms containing the different powers of $\hat{\Psi}_{\perp}^{\dagger}$, we finally obtain

$$
\begin{aligned}
\mathcal{H}|N: \phi\rangle=\sqrt{N}\left(\sum_{\mathbf{r}} \Delta V \chi(\mathbf{r}) \hat{\Psi}^{\dagger}(\mathbf{r})\right)|N-1: \phi\rangle & \\
& +\frac{\sqrt{N(N-1)}}{2}\left(\sum_{\mathbf{r}} \Delta V g_{0} \phi(\mathbf{r}) \phi(\mathbf{r}) \hat{\Psi}_{\perp}^{\dagger}(\mathbf{r}) \hat{\Psi}_{\perp}^{\dagger}(\mathbf{r})\right)|N-2: \phi\rangle
\end{aligned}
$$

with

$$
\chi(\mathbf{r})=\left[h_{0}+\frac{g_{0}(N-1)}{\|\phi\|^{2}}|\phi(\mathbf{r})|^{2}-\frac{g_{0}(N-1)}{2\|\phi\|^{4}} \sum_{\mathbf{r}^{\prime}} \Delta V\left|\phi\left(\mathbf{r}^{\prime}\right)\right|^{4}\right] \phi(\mathbf{r}) .
$$

On the other hand, the variation of a Hartree-Fock state following a variation of $\phi(\mathbf{r})$ can be calculated by replacing $\phi(\mathbf{r})$ by $\phi(\mathbf{r})+d \phi(\mathbf{r})$ in (3) and expanding with the binomial formula. Up to second order in the variation $d \phi(\mathbf{r})$, this leads to

$$
\begin{aligned}
|N: \phi+d \phi\rangle=|N: \phi\rangle+\sqrt{N}\left(\sum_{\mathbf{r}} \Delta V\right. & \left.d \phi(\mathbf{r}) \hat{\Psi}^{\dagger}(\mathbf{r})\right)|N-1: \phi\rangle \\
& +\frac{\sqrt{N(N-1)}}{2}\left(\sum_{\mathbf{r}, \mathbf{r}^{\prime}} \Delta V^{2} d \phi(\mathbf{r}) d \phi\left(\mathbf{r}^{\prime}\right) \hat{\Psi}^{\dagger}(\mathbf{r}) \hat{\Psi}^{\dagger}\left(\mathbf{r}^{\prime}\right)\right)|N-2: \phi\rangle+\ldots
\end{aligned}
$$

In the usual Hartree-Fock mean-field approximation, $\phi$ has a deterministic evolution with $d \phi \propto d t$, so that the last term on the right-hand side of (13) is negligible in the limit $d t \rightarrow 0$ and therefore the last term on the right-hand side of (11) cannot be accounted for. The best that one can do in the mean-field approximation is to set

$$
\left.d \phi(\mathbf{r})\right|_{\mathrm{MF}}=\frac{d t}{i \hbar} \chi(\mathbf{r})
$$

thus recovering a nonlinear Schrödinger equation for $\phi$ of the same form as the usual Gross-Pitaevskii equation [1].

The main idea to recover the exact evolution is to include in $d \phi$ a term $d B$ proportional to $\sqrt{d t}$ :

$$
d \phi(\mathbf{r})=\frac{d t}{i \hbar} \chi(\mathbf{r})+d B(\mathbf{r})
$$

so that the quadratic term in $d \phi$ in (13) is no longer negligible. The price to pay for this is the apparition of an extra term proportional to $\sqrt{d t}$ in (13) which has no counterpart in (11). The trick is to make $d B$ stochastic with a vanishing mean value. Then the average variation of the Hartree-Fock ansatz exactly matches the one required by the many-body Schrödinger equation:

$$
\overline{d|N: \phi\rangle}=\frac{d t}{i \hbar} \mathcal{H}|N: \phi\rangle
$$

provided that the noise term has the following correlation function

$$
\overline{d B(\mathbf{r}) d B\left(\mathbf{r}^{\prime}\right)}=\frac{d t}{i \hbar} \mathcal{Q}_{\mathbf{r}} \mathcal{Q}_{\mathbf{r}^{\prime}}\left[\frac{g_{0}}{\Delta V} \delta_{\mathbf{r}, \mathbf{r}^{\prime}} \phi(\mathbf{r}) \phi\left(\mathbf{r}^{\prime}\right)\right]
$$

where the projector $\mathcal{Q}_{\mathbf{r}}=\mathbf{1}-|\phi\rangle\langle\phi| /\|\phi\|^{2}$ projects orthogonally to $\phi$. The zero mean property of the noise makes the terms proportional to $\sqrt{d t}$ vanish in the averaging over noise. The terms of order three or more in $d \phi$ in the expansion (13) are negligible as compared to $d t$ in the limit $d t \rightarrow 0$. In mathematical terms, this procedure corresponds to assuming that the evolution of $\phi$ is governed by an Ito stochastic differential equation [10. Fig.1 gives a geometrical interpretation of the Monte Carlo sampling of the many-body state vector $|\psi\rangle$ by Hartree-Fock states. The exact many-body state vector at any time $t>0|\psi(t)\rangle$ is equal to the statistical average of Hartree-Fock ansatz:

$$
|\psi(t)\rangle=\langle\mid N: \phi(t)\rangle\rangle
$$




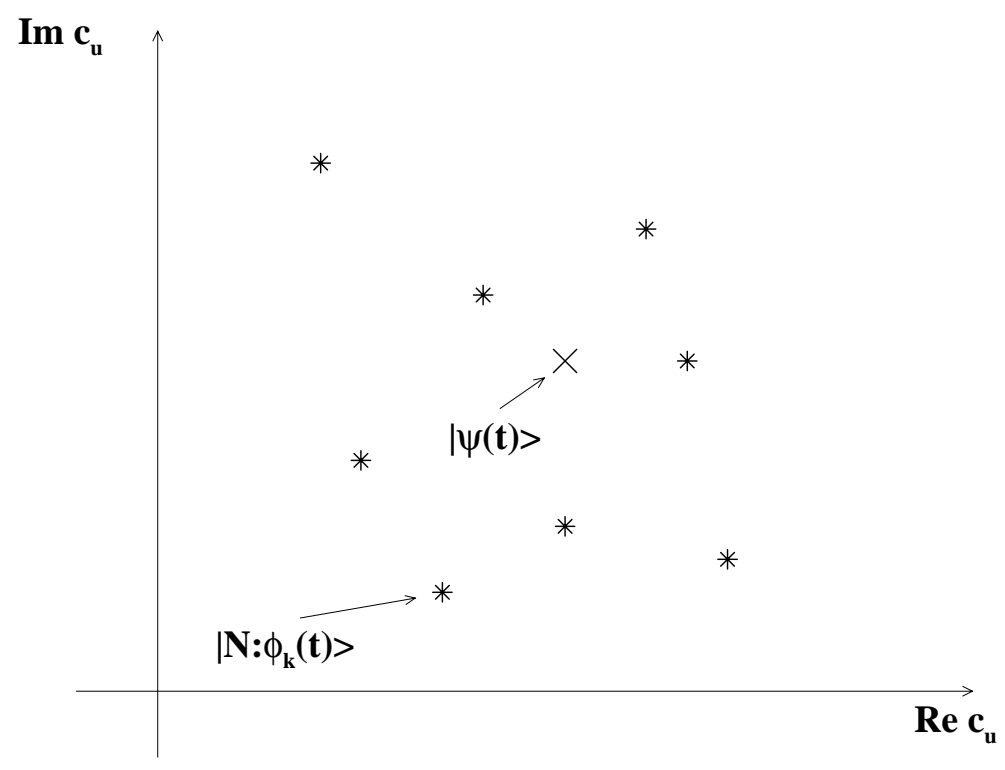

FIG. 1: Schematic view of the Monte Carlo sampling of the exact many-body state vector $|\psi\rangle$ at time $t$ (cross) by a collection of random Hartree-Fock states $\left|N: \phi_{k}\right\rangle$ (stars). In the limit of an infinite number of realizations, the center of mass of the distribution of the stars exactly coincides with the cross. In the $N$-body Hilbert space, $u$ is an arbitrary unit vector and $c_{u}$ is the component along $u$ of $|\psi\rangle$ and of the $\left|N: \phi_{k}\right\rangle$.

provided that this is the case at the initial time $t=0$.

In practice, a noise with the correlation function (17) can be obtained as

$$
d B(\mathbf{r})=\sqrt{\frac{d t g_{0}}{i \hbar V}} \mathcal{Q}_{\mathbf{r}}\left[\phi(\mathbf{r}) \sum_{\mathbf{k}>0}\left(e^{i(\mathbf{k} \cdot \mathbf{r}+\theta(\mathbf{k}))}+\text { c.c. }\right)\right] ;
$$

this expression was already discussed in [4 and is easily generalized to finite range interaction potentials. The index $\mathbf{k}$ is restricted to a half space and to the first Brillouin zone. The $\theta(\mathbf{k})$ 's are random angles uniformly distributed in $[0,2 \pi]$. An alternative choice can be

$$
d B(\mathbf{r})=\sqrt{\frac{d t g_{0}}{i \hbar \Delta V}} \mathcal{Q}_{\mathbf{r}}[\phi(\mathbf{r}) d \xi(\mathbf{r})]
$$

with the $d \xi(\mathbf{r})$ independent zero-mean random variables with $\overline{d \xi(\mathbf{r}) d \xi\left(\mathbf{r}^{\prime}\right)}=\delta_{\mathbf{r}, \mathbf{r}^{\prime}} d t$. In both cases

$$
\overline{\|d B\|^{2}} \leq \frac{g_{0} d t}{\hbar \Delta V}\|\phi\|^{2}
$$

We can then calculate the evolution of the squared norm $\|\phi\|^{2}$ and check that it remains finite at all times:

$$
d\|\phi\|^{2}=\overline{\|d B\|^{2}} \leq \frac{g_{0} d t}{\hbar \Delta V}\|\phi\|^{2},
$$

the absence of terms linear in $d B$ being due to the orthogonality of $d B$ with respect to $\phi$. This leads to

$$
\|\phi\|^{2}(t) \leq\|\phi\|^{2}(0) e^{\frac{g_{0} t}{\hbar \Delta V}}
$$

so that $\phi$ remains finite at all times. The present stochastic wave function reformulation of the bosonic many-body problem is exactly the same as the so-called simple scheme with Fock states introduced in [4] from a different and more general point of view. Among the schemes based on a Hartree-Fock ansatz for the many-body density matrix of the form

$$
\sigma(t)=\left|N: \phi_{1}\right\rangle\left\langle N: \phi_{2}\right|
$$


with $\phi_{1,2}$ evolving according to stochastic differential equations, the simple Fock scheme was shown to be the one which minimizes the growth rate of the statistical variance of $\sigma$ around the exact many-body density matrix $\rho(t)$ :

$$
\operatorname{Var}[\sigma] \equiv\left\langle\|\rho(t)-\sigma(t)\|^{2}\right\rangle=\left\langle\operatorname{Tr}\left[\sigma^{\dagger}(t) \sigma(t)\right]\right\rangle-\operatorname{Tr}\left[\rho(t)^{2}\right]
$$

where $\langle\ldots\rangle$ represents the average over all stochastic realizations. The last term in the above equation, the socalled purity of $\rho$, is constant for Hamiltonian evolutions. If the stochastic wave functions $\phi_{1}$ and $\phi_{2}$ are statistically independent at the initial time $t=0$, they remain so at any later time since their evolutions are independent. The mean squared norm of $\sigma$ is then

$$
\left\langle\operatorname{Tr}\left[\sigma^{\dagger}(t) \sigma(t)\right]\right\rangle=\langle\Delta\rangle^{2}(t)
$$

where

$$
\Delta(t) \equiv\langle N: \phi(t) \mid N: \phi(t)\rangle=\|\phi\|^{2 N}
$$

A geometrical interpretation of $\Delta$ can be put forward in the spirit of fig.1: the mean value of $\Delta$ is related to the mean value of the squared distance in Hilbert space between the Monte Carlo ansatz and the exact many-body state vector

$$
\left.\langle\Delta\rangle-\||\psi\rangle \|_{H}^{2}=\langle\| \mid N: \phi\rangle-|\psi\rangle \|_{H}^{2}\right\rangle
$$

For the present Fock scheme, it follows from (23) that

$$
\langle\Delta\rangle(t) \leq\langle\Delta\rangle(0) e^{N g_{0} t / \hbar \Delta V}
$$

this upper bound was already discussed in 4 . In the model of this paper, the number of modes of the bosonic field is finite, so that the inequality (29) guarantees that the simulation can predict the expectation value of any observable with a finite statistical dispersion. The Monte Carlo statistical variance $\Delta O^{2}$ on the quantum expectation value $\operatorname{Tr}[\rho O]$ of any observable $O$ is indeed limited from above by

$$
\Delta O^{2} \equiv\left\langle|\operatorname{Tr}[\sigma O]|^{2}\right\rangle-\operatorname{Tr}[\rho O]^{2} \leq \operatorname{Tr}\left[O^{2}\right]\left\langle\operatorname{Tr}\left[\sigma^{\dagger} \sigma\right]\right\rangle-\operatorname{Tr}[\rho O]^{2}
$$

where the traces are taken in the $N$-particle subspace and the trace $\operatorname{Tr}\left[O^{2}\right]$ is a finite quantity for a finite number of modes.

This formalism can be extended to the imaginary-time evolution [1], which allows one to sample in an exact way the thermal equilibrium state at a given temperature $T$ :

$$
\rho=e^{-\mathcal{H} / k_{B} T}
$$

This requires solving the imaginary-time evolution equation

$$
\frac{d}{d \tau} \rho=-\frac{1}{2}\{\mathcal{H}, \rho\}
$$

for a "time" $\tau$ going from 0 to $\beta=1 / k_{B} T$. The use of the projector onto the $N$ particle subspace $\mathcal{P}_{N}$ as the initial condition allows one to calculate the physical quantities in the canonical ensemble, i.e. for a fixed number $N$ of particles. As we shall prove in full detail in the next section, the projector operator $\mathcal{P}_{N}$ has a simple expression in terms of Hartree-Fock states.

The correct imaginary-time evolution of the Hartree-Fock state $|N: \phi\rangle$ is recovered if the stochastic wave function $\phi(\mathbf{r})$ evolves according to

$$
d \phi(\mathbf{r})=-\frac{d \tau}{2}\left[h_{0}+\frac{g_{0}(N-1)}{\|\phi\|^{2}}|\phi(\mathbf{r})|^{2}-\frac{g_{0}(N-1)}{2\|\phi\|^{4}} \sum_{\mathbf{r}^{\prime}} \Delta V\left|\phi\left(\mathbf{r}^{\prime}\right)\right|^{4}\right] \phi(\mathbf{r})+d B(\mathbf{r})
$$

with a noise term $d B(\mathbf{r})$ equal to

$$
d B(\mathbf{r})=i \sqrt{\frac{d \tau g_{0}}{2 V}} \mathcal{Q}_{\mathbf{r}}\left[\phi(\mathbf{r}) \sum_{\mathbf{k}>0}\left(e^{i(\mathbf{k} \cdot \mathbf{r}+\theta(\mathbf{k}))}+\text { c.c. }\right)\right] .
$$

We have recently applied this method to study the probability distribution of the number of condensate particles in a 1D trapped Bose gas for temperatures both above and below the transition temperature [12]. 


\section{PROOF OF (OVER)-COMPLETENESS OF THE HARTREE-FOCK STATES}

In the previous section we have shown how the $N$-boson time-dependent problem can be exactly reformulated in terms of the stochastic evolution of single particle wave functions; in particular, the thermal equilibrium state in the canonical ensemble can be obtained by means of an imaginary-time evolution with the projector $\mathcal{P}_{N}$ as the initial state. Here, we shall prove that the projector $\mathcal{P}_{N}$ has a simple expression in terms of Hartree-Fock states and that any state vector can be expanded onto a set of Hartree-Fock states. This ensures that the identity (18) can be satisfied at time $t=0$ for any initial many-body state vector $|\psi(0)\rangle$.

It is a well-known fact of quantum optics [13] that the Glauber coherent states form an overcomplete basis of the Hilbert space of an harmonic oscillator. In the general multi-mode case, this property can be expressed as

$$
\left.\mathbf{1}=\frac{1}{\pi^{M}} \int \mathcal{D} \phi \mid \text { Glaub : } \phi\right\rangle\langle\text { Glaub : } \phi|
$$

where the Glauber coherent state is defined as usual as

$$
\mid \text { Glaub : } \phi\rangle=e^{-\|\phi\|^{2} / 2} e^{\sum_{\mathbf{r}} \Delta V \phi(\mathbf{r}) \hat{\Psi}^{\dagger}(\mathbf{r})}|0\rangle .
$$

and 1 is the identity on the bosonic subspace of totally symmetric wave functions. The integration in (35) is performed over the $2 M$ dimensional space of complex-valued functions defined on the $M$-point lattice:

$$
\mathcal{D} \phi=\prod_{\mathbf{r}} d \operatorname{Re} \phi(\mathbf{r}) d \operatorname{Im} \phi(\mathbf{r}) .
$$

Using the well-known formula

$$
\left.\mathcal{P}_{N} \mid \text { Glaub }: \phi\right\rangle=\frac{1}{\sqrt{N !}} e^{-\|\phi\|^{2} / 2}|N: \phi\rangle
$$

to project onto the $N$ particle subspace, an explicit expression for $\mathcal{P}_{N}$ in terms of Hartree-Fock states can be obtained

$$
\left.\mathcal{P}_{N}=\mathcal{P}_{N} \mathbf{1} \mathcal{P}_{N}=\frac{1}{\pi^{M}} \int \mathcal{D} \phi \mathcal{P}_{N} \mid \text { Glaub }: \phi\right\rangle\left\langle\text { Glaub }: \phi\left|\mathcal{P}_{N}=C \int_{1} \mathcal{D} \hat{\phi}\right| N: \hat{\phi}\right\rangle\langle N: \hat{\phi}| .
$$

The constant $C$ is equal to

$$
C \equiv \frac{1}{N ! \pi^{M}} \int d\|\phi\|\|\phi\|^{2 N+2 M-1} e^{-\|\phi\|^{2}}=\frac{(M+N-1) !}{2 \pi^{M} N !} .
$$

The normalized wave function $\hat{\phi}(\mathbf{r})$ is defined as $\hat{\phi}(\mathbf{r})=\phi(\mathbf{r}) /\|\phi\|$ and the integration over $\hat{\phi}$ is performed on the unit sphere $\|\hat{\phi}\|^{2}=1$ according to the measure $\mathcal{D} \hat{\phi}$ defined as

$$
\mathcal{D} \phi=\|\phi\|^{2 M-1} d\|\phi\| \mathcal{D} \hat{\phi} .
$$

As discussed in [11, the expression (39) is the starting point for an exact numerical calculation of the thermodynamical equilibrium properties of the interacting Bose gas.

Completeness of the set of Hartree-Fock states is an immediate consequence of the result (39): any state vector $|\psi\rangle$ belonging to the $N$ particle subspace can in fact be expanded over Hartree-Fock states according to

$$
|\psi\rangle=\mathcal{P}_{N}|\psi\rangle=C \int_{1} \mathcal{D} \hat{\phi}\langle N: \hat{\phi} \mid \psi\rangle|N: \hat{\phi}\rangle .
$$

As required for practical implementation of the Fock scheme, the weight factor can be made real and positive for all the $\hat{\phi}$ just by reabsorbing the phase of $\langle N: \hat{\phi} \mid \psi\rangle$ into that of the wave function $\hat{\phi}$ :

$$
|\psi\rangle=C \int_{1} \mathcal{D} \hat{\phi}|\langle N: \hat{\phi} \mid \psi\rangle||N: r(\hat{\phi}) \hat{\phi}\rangle
$$

with the phase factor $r(\hat{\phi})$ defined according to

$$
r(\hat{\phi})^{N}=\frac{\langle N: \hat{\phi} \mid \psi\rangle}{|\langle N: \hat{\phi} \mid \psi\rangle|} .
$$


Since the scalar product of two Hartree-Fock states is equal to

$$
\left\langle N: \phi_{2} \mid N: \phi_{1}\right\rangle=\left\langle\phi_{2} \mid \phi_{1}\right\rangle^{N},
$$

this family of states is not an orthonormal basis, but rather an overcomplete set.

\section{STOCHASTIC SCHEMES WITH COHERENT STATES: AN INFINITE STATISTICAL UNCERTAINTY}

\section{The Bargmann scheme}

The derivation of the Fock scheme can be reproduced for the case of unnormalized coherent states, the so-called Bargmann states:

$$
|\operatorname{Barg}: \phi\rangle=\Pi_{0} e^{\sum_{\mathbf{r}} \Delta V \phi(\mathbf{r}) \hat{\Psi}^{\dagger}(\mathbf{r})}|0\rangle,
$$

where $\phi$ is a stochastic dynamical variable and $\Pi_{0}$ is a time-independent amplitude. Thanks to (35), one can show that any initial state vector $|\psi(0)\rangle$ can be obtained as a statistical average of Bargmann states of the form (46); the initial value of both $\phi$ and $\Pi_{0}$ can then vary randomly from one Monte Carlo realization to the other.

The exact time-evolution given by the many-body Hamiltonian (2) can be shown to be exactly recovered if the field amplitude $\phi(\mathbf{r})$ evolves according to

$$
d \phi(\mathbf{r})=\frac{d t}{i \hbar} h_{0} \phi(\mathbf{r})+d B(\mathbf{r})
$$

with a noise correlation function equal to

$$
\overline{d B(\mathbf{r}) d B\left(\mathbf{r}^{\prime}\right)}=\frac{d t}{i \hbar} \frac{g_{0}}{\Delta V} \delta_{\mathbf{r}, \mathbf{r}^{\prime}} \phi(\mathbf{r}) \phi\left(\mathbf{r}^{\prime}\right) .
$$

In practice, we take the following implementation of the noise which minimizes the squared norm of $d B$ [ [4]:

$$
d B(\mathbf{r})=\sqrt{\frac{d t g_{0}}{i \hbar V}} \phi(\mathbf{r}) \sum_{\mathbf{k}>0}\left(e^{i(\mathbf{k} \cdot \mathbf{r}+\theta(\mathbf{k}))}+\text { c.c. }\right),
$$

where $\mathbf{k}$ is restricted to a half space and to the first Brillouin zone. Within the framework of [ $\llbracket$, this Bargmann scheme recovers one of the possible solutions of the consistency equations in the case of a coherent state ansatz, namely the one with a constant amplitude $\Pi(t)$ at all times.

Here we shall prove that, contrary to the scheme with Fock states, the scheme (47,48) has an infinite statistical uncertainty for any time $t>0$, in a sense to be precised below. As in the section with Fock states we introduce

$$
\Delta(t) \equiv\langle\operatorname{Barg}: \phi(t) \mid \operatorname{Barg}: \phi(t)\rangle=\left|\Pi_{0}\right|^{2} e^{\|\phi(t)\|^{2}} .
$$

As initial state at $t=0$ we take for simplicity a pure Bargmann state $|\operatorname{Barg}: \phi(0)\rangle$ with in average $\|\phi(0)\|^{2}=N$ particles. $\Pi_{0}$ is taken as $\exp (-N / 2)$, so that the initial state is normalized to unity. We now show that the mean value of $\Delta(t)$ is infinite for any $t>0$.

From (47) and (48), it follows that the evolution of $X(t)=\|\phi(t)\|^{2}$ is described by

$$
d X=\langle d B \mid d B\rangle+\langle d B \mid \phi\rangle+\langle\phi \mid d B\rangle=\frac{g_{0}}{\hbar \Delta V} X d t+d \eta
$$

where

$$
d \eta=\langle d B \mid \phi\rangle+\langle\phi \mid d B\rangle
$$

is a zero-mean noise term and its variance is equal to

$$
\overline{d \eta^{2}}=\overline{(\langle d B \mid \phi\rangle+\langle\phi \mid d B\rangle)^{2}}=\overline{\langle\phi \mid d B\rangle^{2}}+\overline{\langle d B \mid \phi\rangle^{2}}+2 \overline{\langle d B \mid \phi\rangle\langle\phi \mid d B\rangle} .
$$


Inserting in this expression the noise correlation function (48), the first two terms on the right-hand side of (53) cancel. For the remaining term, a lower bound can be obtained by using the explicit expression (49) for the noise and applying the Schwartz inequality ${ }^{2}$ :

$$
\overline{d \eta^{2}}=2 \overline{\langle d B \mid \phi\rangle\langle\phi \mid d B\rangle}=\frac{2 g_{0} d t}{\hbar} \sum_{\mathbf{r}} \Delta V|\phi(\mathbf{r})|^{4} \geq \frac{2}{M} \frac{g_{0}}{\hbar \Delta V} X^{2} d t
$$

as in the previous sections, $M$ is the number of lattice points.

From (51), a lower bound on the increase of the moments of $\left\langle X^{n}\right\rangle(t)$ can be obtained:

$$
\begin{aligned}
d\left\langle X^{n}\right\rangle=n\left\langle X^{n-1} d X\right\rangle+\frac{n(n-1)}{2}\left\langle X^{n-2} d X^{2}\right\rangle=\frac{g_{0} d t}{\Delta V \hbar} n\left\langle X^{n}\right\rangle+\frac{n(n-1)}{2}\left\langle X^{n-2} d \eta^{2}\right\rangle \geq & \\
& \geq \frac{g_{0} d t}{\Delta V \hbar}\left[n\left\langle X^{n}\right\rangle+\frac{n(n-1)}{M}\left\langle X^{n}\right\rangle\right]
\end{aligned}
$$

and therefore on the moments themselves:

$$
\left\langle X^{n}\right\rangle(t) \geq\left\langle X^{n}\right\rangle(0) e^{\gamma(n) \tau}=N^{n} e^{\gamma(n) \tau} .
$$

Here we have set $\tau=g_{0} t / \Delta V \hbar$ and

$$
\gamma(n)=n+n(n-1) / M
$$

This leads to the lower bound for the mean value of $\Delta(t)$ :

$$
\langle\Delta\rangle(t)=\left|\Pi_{0}\right|^{2}\langle\exp X\rangle \geq\left|\Pi_{0}\right|^{2} \sum_{n=0}^{\infty} \frac{1}{n !} N^{n} e^{\gamma(n) \tau} .
$$

As the ratio of two successive elements of the series tends to infinity in the limit $n \rightarrow \infty$ for any $t>0$, this results in an infinite value of $\langle\Delta\rangle(t)$ and therefore of the variance of the statistical error on the many-body density operator.

The same result could have been obtained by explicitly calculating the probability distribution for $X$ at a given time $t$ : as the evolution of the random variable $Y=\ln (X)$ is a simple diffusive motion in presence of a constant drift term, its probability distribution $P(Y, t)$ has a Gaussian shape at all times $t$. By going back to the original variable $X$ by means of $P(X, t)=\frac{d Y}{d X} P(Y, t)$, the distribution of $X$ is found to be equal to

$$
P(X, t)=\frac{1}{X}\left(\frac{M}{4 \pi \tau}\right)^{1 / 2} e^{-\frac{M}{4 \tau}\left[\ln (X / N)-\left(1-\frac{1}{M}\right) \tau\right]^{2}}
$$

It follows that the average of the exponential $\exp (X)$ does not converge at $X=+\infty$ and therefore that the average of $(50)$ is indeed infinite.

One might wonder if the same conclusion holds for other indicators of the magnitude of the statistical error. For example, one could consider the mean value of the distance $\delta$ between the Monte Carlo ansatz $\mid$ Barg : $\phi\rangle$ and the exact many-body state vector $|\psi\rangle$ :

$$
\delta=\||\operatorname{Barg}: \phi\rangle-|\psi\rangle \|_{H}
$$

where $\|\ldots\|_{H}$ is the usual norm in the many-body Hilbert space. As

$$
\||\operatorname{Barg}: \phi\rangle\left\|_{H} \leq\right\||\operatorname{Barg}: \phi\rangle-|\psi\rangle\left\|_{H}+\right\||\psi\rangle \|_{H}
$$

the mean distance $\langle\delta\rangle$ is bounded from below by

$$
\langle\delta\rangle \geq\left\langle\Delta^{1 / 2}\right\rangle-\||\psi\rangle \|_{H}
$$

\footnotetext{
${ }^{2}$ For a fixed value of the norm of the field $\|\phi\|$, the sum $\sum_{\mathbf{r}}|\phi(\mathbf{r})|^{4}$ is minimum if the density $|\phi(\mathbf{r})|^{2}$ is a constant, which implies $\sum_{\mathbf{r}} \Delta V|\phi(\mathbf{r})|^{4} \geq\|\phi\|^{4} / M \Delta V$.
} 
By a straightforward generalization of (58), we find that the mean value of $\Delta^{1 / 2}$ is infinite for any $t>0$, so that the mean value of $\delta$ is infinite. From the explicit form of the probability distribution (59) one can also check that the same result holds for any moment $\left\langle\delta^{\alpha}\right\rangle$ of the distance $\delta$, with an arbitrary real exponent $\alpha>0$.

These results nicely demonstrate the difference between the convergence of the stochastic process for the field amplitude $\phi$ and for the many-body ansatz. Since the deterministic term (47) is norm-conserving and the noise term (48) does not grow faster than $\|\phi\|$, one can apply a regularity theorem which guarantees that no finite time divergences can occur in the random dynamics of the field amplitude $\phi$ and that all the moments of the stochastic wave function are finite (cf. [10, chap. 4.3] and [14, chap.6-7]). However, the statistical uncertainty is still divergent because the Bargmann ansatz involves an exponential function of the field amplitude $\phi(\mathbf{r})$ and not only a polynomial one as was the case with Fock states 柾.

\section{The simple scheme with coherent states}

In the previous subsection we showed that the statistical uncertainty for a simulation performed according to the Bargmann scheme is infinite. In [4] we have identified among all the schemes based on coherent states the one (the so-called simple scheme) which minimizes the growth rate of the variance of the statistical error on the many-body density operator. Here we shall show that for any finite time the statistical uncertainty is infinite also for the simple scheme, similarly to the case of the Bargmann scheme.

The scheme denoted in 迎 as the simple scheme with coherent states corresponds to a slightly more general ansatz than (46):

$$
|\operatorname{coh}:(\phi, \Pi)\rangle=\Pi e^{\sum_{\mathbf{r}} \Delta V \phi(\mathbf{r}) \hat{\Psi}^{\dagger}(\mathbf{r})}|0\rangle
$$

where the amplitude $\Pi(t)$ is now a stochastically evolving dynamical variable. The field amplitude $\phi$ now solves the Ito differential stochastic equation

$$
d \phi(\mathbf{r})=\frac{d t}{i \hbar}\left[h_{0}+g_{0}|\phi(\mathbf{r})|^{2}\right] \phi(\mathbf{r})+d B(\mathbf{r})
$$

with exactly the same noise term $d B$ as in the Bargmann scheme, see (49). The evolution of the amplitude $\Pi$ is given by

$$
d \Pi=-\Pi \sum_{\mathbf{r}} \Delta V \phi^{*}(\mathbf{r}) d B(\mathbf{r})=-\Pi\langle\phi \mid d B\rangle .
$$

The deterministic term in $d \phi$ includes the usual mean field contribution. As it remains norm conserving, the results previously proved for the distribution and the moments of $X(t)=\|\phi\|^{2}$ for the Bargmann scheme still hold for the present scheme. In particular, the stochastic differential equation (64) has a well defined solution according to the regularity theorem of [10, 14]. Setting $\Pi=\exp S$, equation (65) can be turned into

$$
d S=-\langle\phi \mid d B\rangle-\frac{1}{2}\langle\phi \mid d B\rangle^{2} .
$$

Using the explicit expression for the correlation function of $d B$, this expression simplifies to

$$
d S=-\langle\phi \mid d B\rangle-\frac{g_{0} d t}{2 i \hbar} \sum_{\mathbf{r}} \Delta V|\phi(\mathbf{r})|^{4} .
$$

This equation for $S$ can be integrated by quadrature proving the existence of a regular solution to (65).

As in the previous section we quantify the statistical spread in the simulation with

$$
\Delta(t) \equiv\langle\operatorname{coh}:(\phi, \Pi) \mid \operatorname{coh}:(\phi, \Pi)\rangle .
$$

As the initial state at $t=0$, we take for simplicity a pure coherent state $|\operatorname{coh}:(\phi(0), \Pi(0))\rangle$ with on average $\|\phi(0)\|^{2}=N$ particles and an amplitude $\Pi(0)=\exp (-N / 2)$, so that the state vector is normalized to unity.

The time evolution of $\Delta(t)$ is given by [4]:

$$
d \Delta=\|d B\|^{2} \Delta=\frac{g_{0}}{\hbar \Delta V} X \Delta d t .
$$


Because of the nonlinear term proportional to $X(t) \Delta(t)$ in (69), the coupled system of (69) and (51) does not fulfill all the hypothesis of the regularity theorem [10, chap. 4.3] and therefore we can expect singular behaviors.

In terms of the rescaled time $\tau=g_{0} t / \Delta V \hbar$, the stochastic equation of motion for $X(\tau)$ and $\Delta(\tau)$ can be rewritten as

$$
\begin{aligned}
& d X=X d \tau+d \eta \\
& d \Delta=X \Delta d \tau,
\end{aligned}
$$

where the zero-mean noise $d \eta$ satisfies, as in the previous section:

$$
\overline{d \eta^{2}} \geq \frac{2}{M} X^{2} d \tau
$$

It follows from (71) that

$$
\Delta(t)=\exp \left(\int_{0}^{\tau} d \tau^{\prime} X\left(\tau^{\prime}\right)\right)
$$

and therefore its mean value can be written as the series

$$
\langle\Delta\rangle(\tau)=1+\sum_{n=1}^{\infty} D_{n}(\tau)
$$

whose coefficients $D_{n}(\tau)$ are given by integrals of the form

$$
D_{n}(\tau)=\int_{\tau>\tau_{n}>\tau_{n-1}>\ldots>\tau_{2}>\tau_{1}>0}^{d \tau_{n} d \tau_{n-1} \ldots d \tau_{2} d \tau_{1}}\left\langle X\left(\tau_{n}\right) X\left(\tau_{n-1}\right) \ldots X\left(\tau_{2}\right) X\left(\tau_{1}\right)\right\rangle .
$$

Using the regression theorem of stochastic analysis [10] and the fact that all moments (56) of $X$ are increasing functions of $\tau$, it is easy to see that

$$
\left\langle X^{k}(\tau) X\left(\tau_{n-k}\right) X\left(\tau_{n-k-1}\right) \ldots X\left(\tau_{2}\right) X\left(\tau_{1}\right)\right\rangle
$$

is itself an increasing function of $\tau$ for any $1 \leq k \leq n$ and for $\tau \geq \tau_{n-k} \geq \tau_{n-k-1} \geq \ldots \geq \tau_{1} \geq 0$. This implies that

$$
\begin{aligned}
\left\langle X\left(\tau_{n}\right) X\left(\tau_{n-1}\right) X\left(\tau_{n-2}\right) \ldots X\left(\tau_{1}\right)\right\rangle & \geq \\
& \geq\left\langle X\left(\tau_{n-1}\right)^{2} X\left(\tau_{n-2}\right) \ldots X\left(\tau_{1}\right)\right\rangle \geq\left\langle X\left(\tau_{n-2}\right)^{3} \ldots X\left(\tau_{1}\right)\right\rangle \geq \ldots \geq\left\langle X\left(\tau_{1}\right)^{n}\right\rangle \geq N^{n} e^{\gamma(n) \tau_{1}} .
\end{aligned}
$$

For $\tau>0$, we then have

$$
D_{n}(\tau) \geq \frac{N^{n} e^{\gamma(n) \tau}}{(n-1) ! \gamma(n)^{n}} \int_{0}^{\gamma(n) \tau} e^{-w} w^{n-1} d w .
$$

The integral in $w$ can be explicitly performed and for values of $n$ sufficiently large that $\gamma(n) \tau \geq 1$, it turns out to be bounded from below as follows:

$$
\int_{0}^{\gamma(n) \tau} e^{-w} w^{n-1} d w=(n-1) !\left[1-e^{-\gamma(n) \tau} \sum_{j=0}^{n-1} \frac{(\gamma(n) \tau)^{j}}{j !}\right] \geq(n-1) !\left[1-n(\gamma(n) \tau)^{n-1} e^{-\gamma(n) \tau}\right]
$$

which means that

$$
D_{n} \geq \frac{N^{n} e^{\gamma(n) \tau}}{\gamma(n)^{n}}\left[1-n(\gamma(n) \tau)^{n-1} e^{-\gamma(n) \tau}\right] \equiv E_{n}
$$

Since

$$
\lim _{n \rightarrow \infty} \frac{E_{n+1}}{E_{n}} \equiv+\infty
$$

the infinite sum of the $E_{n}$ is infinite as well as the sum of the $D_{n}$ which appears in (74). This proves that the variance of the statistical error on the many-body density matrix is indeed infinite also in the case of the simple scheme with coherent states.

As for the Bargmann scheme, this result can be generalized to other indicators of the statistical error, e.g. the distance $\delta$ between the Monte Carlo ansatz and the exact state vector

$$
\delta=\||\operatorname{coh}:(\phi, \Pi)\rangle-|\psi\rangle \|_{H}
$$

has an infinite mean for $t>0$. 


\section{CONCLUSION}

We have shown that the simple Fock scheme can be rederived in a very elementary way using the Schrödinger equation for the state vector rather than the Liouville equation for the many-body density operator. Such a derivation is inspired from the derivation for fermions used in 8 . We have also given a proof of completeness of the Hartree-Fock state ansatz more detailed than in [4].

For the two schemes with coherent states considered in this paper, we have shown that the absolute value of the statistical error on the many-body state vector has an infinite mean for any finite evolution time. This negative result has been obtained by analytical means for a continuous time evolution. Of course, any actual numerical calculation is performed with a finite time step $d t$ so that any given time $t$ is reached with a finite number of steps. As the noise (49) is bounded from above, this imposes an upper limit to the maximum value of the statistical error which can be achieved by any realization and therefore its mean value remains finite in the numerics. This is why this problem of the simple coherent scheme was not discovered in the numerical simulations of $[$ ].

We acknowledge useful discussions with Crispin Gardiner, Karen Kheruntsyan, Peter Drummond, Slava Yukalov, Reinhold Walser, Eugene Zaremba, Carlos Lobo, Dimitri Gangardt, Alice Sinatra, Philippe Chomaz and Olivier Juillet. I.C. acknowledges a Marie Curie grant from the EU under contract number HPMF-CT-2000-00901. Laboratoire Kastler Brossel is a Unité de Recherche de l'École Normale Supérieure et de l'Université Paris 6, associée au CNRS.

* Electronic address: Yvan.Castin@lkb.ens.fn

[1] Dalfovo F., Giorgini S., Pitaevskii L., and Stringari S., 1999, Rev. Mod. Phys. 71, 463.

[2] Drummond P. D. and Gardiner C. W., 1980, J. Phys. A 13, 2353.

[3] Gilchrist A., Gardiner C. W., and Drummond P. D., 1997, Phys. Rev. A 55, 3014.

[4] Carusotto I., Castin Y., and Dalibard J., 2001, Phys. Rev. A 63, 023606.

[5] Plimak L. I., Olsen M. K., Fleischhauer M., and Collett M. J., 2001, Europhys. Lett. 56, 372.

[6] Deuar P. and Drummond P. D., 2002, preprints quant-ph/0203108 and quant-ph/0203025.

[7] Plimak L. I., Collett M. J., and Olsen M. K., 2001, Phys. Rev. A 64, 063409.

[8] Juillet O. and Chomaz Ph., 2002, Phys. Rev. Lett. 88, 142503.

[9] Bouchaud J.-P. and Georges A., 1990, Phys. Rep. 195, 127; Bardou F., Bouchaud J.-P., Aspect A., and Cohen-Tannoudji C., 2002, Lévy statistics and laser cooling: How rare events bring atoms to rest (Cambridge University Press, Cambridge).

[10] Gardiner C. W., 1983, Handbook of stochastic methods (Springer-Verlag, Berlin).

[11] Carusotto I. and Castin Y., 2001, J. Phys. B 34, 4589.

[12] Carusotto I. and Castin Y., 2002, preprint cond-mat/0206211.

[13] Walls D. F. and Milburn G. J., 1994, Quantum Optics (Springer-Verlag, Berlin).

[14] Arnold L., Stochastic Differential Equations; theory and applications, 1974, (Wiley, New York). 